\title{
A Novel Method for Manufacturing Polymer/Metal Hybrid Structures
}

\author{
M. Altan* AND B. YAVuZ \\ Department of Mechanical Engineering, Ylldı Technical University, Istanbul, Turkey
}

\begin{abstract}
In this study, a method for manufacturing polymer/metal hybrid structure is presented in which traditional plastic injection machine was converted to plastic injection forming (PIF). In this method, deforming of the metal and adhering the metal to the polymer is done in one step. In the experimental study, a special mold within rectangular cavity was used during PIF process. Aluminum plates of different thicknesses were used as the metal parts of the structure. The injected polymers were pure polystyrene and elastomer-added polystyrene. The deformability of the $\mathrm{Al}$ plates was examined under different injection pressures. In order to provide adhesion between metal and polymer, an adhesive of elastomeric nature was applied on the metal plate prior to injection molding. Bending test was applied to determine the flexural strength and maximum deflection of the polymer/metal hybrid structures. The obtained results were satisfactorily acceptable in improving the method for designing and manufacturing polymer/metal hybrid structures in one step.
\end{abstract}

DOI: 10.12693/APhysPolA.129.639

PACS/topics: 61.41.+e, 68.47.De

\section{Introduction}

Polymers and metals have been used in different fields of industry. Each of them has different advantages such as better mechanical strength of metal parts and lightness of polymers. When these two classes of materials are integrated, a superior structure is possible to obtain. The hybrid structures of polymer and metal can be found especially in automotive or aircraft industry for noise or vibration damping components $[1,2]$. Polymer/metal hybrid structures induce the demand of assembly of them in different ways. The primitive method has been used for many years in which polymer and metal structures are assembled by an adhesive. For this process, a good surface treatment before adhesive application is needed. Furthermore, the geometrical shape of the polymer and metal components should be very compatible to have a combined structure having high mechanical strength. In most cases, metal part and polymeric part are fabricated separately by metal forming processes and plastic injection molding. Then, they are joined by adhesion bonding. Another method to obtain polymer/metal hybrid structures is conventional injection molding with metal inserts. Plastic plugs are good example for this method.

Due to the developing technology, it has been realized that polymer/metal hybrid structures need new technologies including almost one step manufacturing in a fully optimized assembly line for metal and polymer components. Primitive methods are not sufficient to meet the geometrical accuracy or they bring large degree of manual labor within cumbersome manufacturing.

*corresponding author; e-mail: meksi@yildiz.edu.tr
Therefore, a conventional process, plastic injection molding is modified for manufacturing plastics covered with metal plates [1-6]. Dimensional tolerance of both polymeric and metal section is provided by this method in one step, which is called polymer injection forming (PIF).

The basic principle of the PIF process was not developed for obtaining polymer/metal structures. Initially, it was developed for deforming sheets under viscous pressure $[4,7]$ and then it was converted to a macro composite manufacturing method by some additional processes before injection forming, for improving the adhesion between polymer and metal [8-10]. In this paper, polymer injection forming is presented. Bending test of the manufactured polymer/metal structures was carried out to determine their mechanical strength. The effect of injection pressure was investigated to examine the deformability of the metal plate.

\section{Materials and methods 2.1. Materials}

Aluminum metal plates (1100) with length and width of $115 \mathrm{~mm}$ and $75 \mathrm{~mm}$, respectively, were used in experiments. Two different thicknesses of $0.5 \mathrm{~mm}$ and $1.5 \mathrm{~mm}$ were tested. Polystyrene (PS) (LGH-306, LG Polymers Inc.) was used as the polymer material. In the experimental study, besides the usage of pure PS, an elastomer, maleic anhydride grafted styrene-ethylenebutylene-styrene (SEBS-g-MA)-added polystyrene was also used.

\subsection{Surface preparation and adhesive application}

Aluminum surfaces were sand-papered with 3M Diapad to increase adhesion between polymer and metal. Then samples were washed with alkaline soap, rinsed in acetone and in distilled water. Before putting aluminium plate on the die cavity, a primer of elastomeric nature (Metsan Inc.) was applied on the plate. 


\subsection{Injection molding}

A 40 ton injection molding machine was used. The injection pressure was changed between $30 \mathrm{MPa}$ and $60 \mathrm{MPa}$ and the difference of the deformability of the aluminum plates was investigated, because of the critical importance of injection pressure for deforming of the metal plate. The other injection parameters were kept constant.

The die in the injection molding machine had a rectangular shape with dimensions of $100 \times 75 \times 4.5 \mathrm{~mm}^{3}$. The schematic picture of the PIF process is given in Fig. 1. Pure polystyrene and elastomer-added polystyrene were injected, respectively.

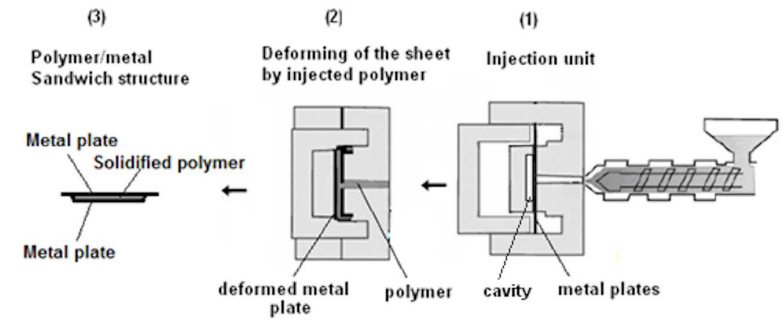

Fig. 1. Schematic picture of polymer injection forming (PIF) process.

\subsection{Bending test}

In order to measure the flexural strength and deflection of the sandwich structures, bending test was applied on Universal Tensile Testing Machine (Zwick 1455). The velocity of the test was $5 \mathrm{~mm} / \mathrm{min}$. The flexural test specimens were prepared by cutting from polymer/metal hybrid structure by water jet under pressure of 3400 bar.

\section{Results and discussion}

In order to see the formability of the plates by polymer injection, aluminum plates were first deformed without application of an adhesive. Undeformed aluminum plate was put onto the cavity as shown in Fig. 1 and then polymer injection forming was applied. In order to obtain the optimal deformed shape of the $\mathrm{Al}$, different injection pressures were experimented. The formability of the metals tested at different values of the injection pressure is given in Fig. 2. Pressure of 30 bar and 40 bar was

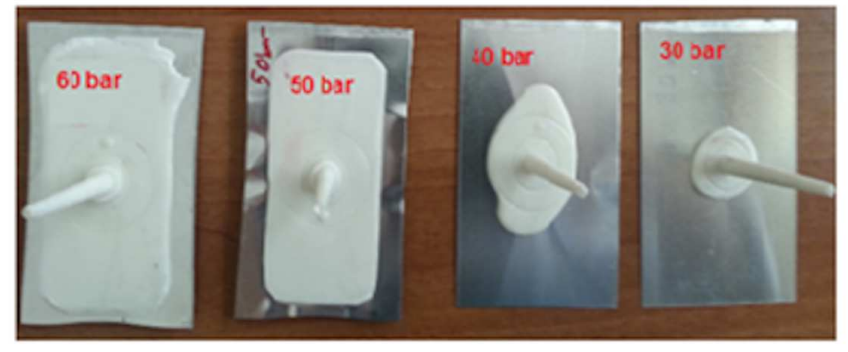

Fig. 2. The formability of the $\mathrm{Al}$ plates tested at different values of the injection pressure. insufficient for deforming the $\mathrm{Al}$ plates. As the pressure increased, the formability of the metal plate increased. On the other hand, at $60 \mathrm{MPa}$, tearing on the plate was observed around the corner where the highest thinning of the material has occurred, as shown in Fig. 3.

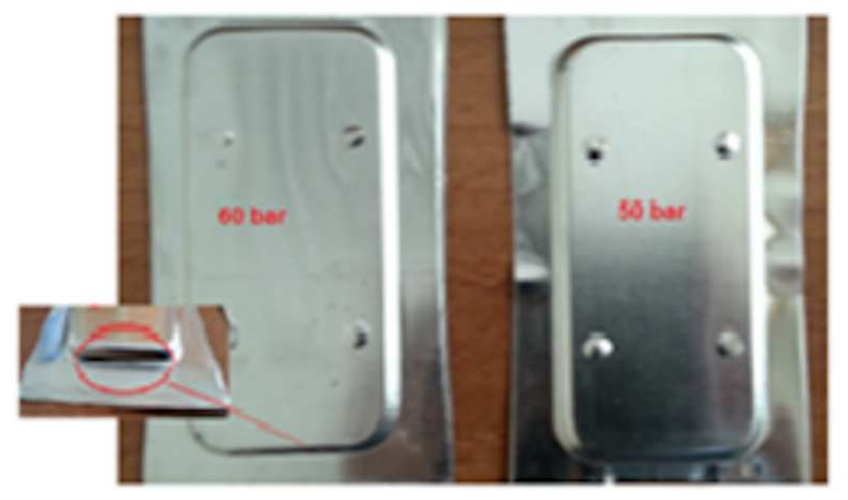

Fig. 3. Tearing effect of pressure.

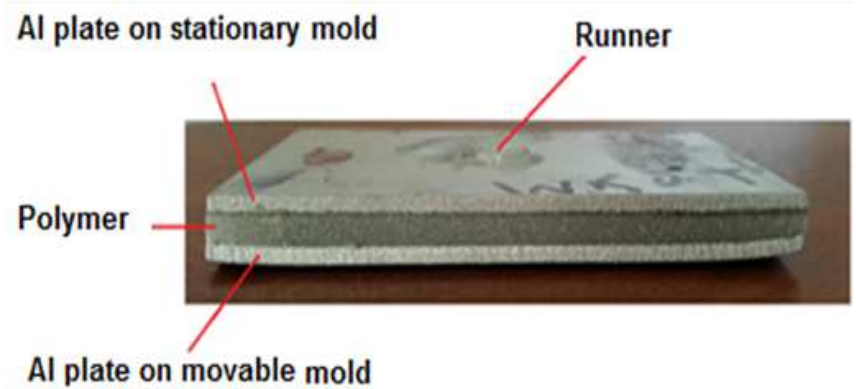

Fig. 4. Cross-section of the hybrid structure.

When thickness of aluminum plate was raised to $1.5 \mathrm{~mm}$, tearing effect was solved and sufficient deforming was realized. The cross section of the obtained hybrid structure from PIF process is given in Fig. 4. Bending test specimens (Fig. 4) of the hybrid structure were cut by water jet. The results of the bending test are reported in Table I.

TABLE I

Bending test results of polymer/metal hybrid structures.

\begin{tabular}{c|c|c}
\hline \hline $\begin{array}{c}\text { Cores of the hybrid } \\
\text { structures }\end{array}$ & $\begin{array}{c}\text { Flexural } \\
\text { strength } \\
\text { [Mpa] }\end{array}$ & $\begin{array}{c}\text { Deflection } \\
\text { at maximum } \\
\text { force [mm] }\end{array}$ \\
\hline PS & 26.95 & 3.93 \\
PS/SEBS-g-MA/\%5 & 34.78 & 6.33 \\
PS/SEBS-g-MA/\%15 & 35.39 & 6.65
\end{tabular}

The polymeric core with pure polystyrene gave less deflection due to its harder structure, compared to that of polystyrene with elastomeric material, SEBS. As a result, a crack has occurred in the core of pure polystyrene, as shown in Fig. 5. 

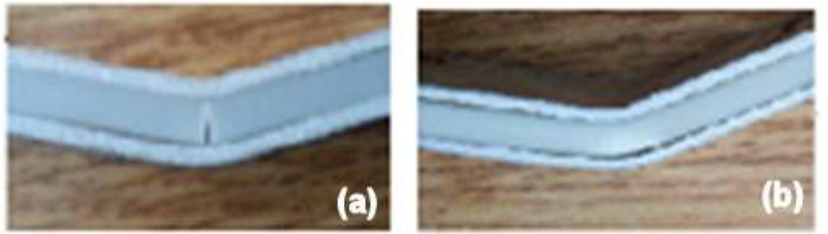

Fig. 5. Photographs of the specimen after bending test. (a) Pure PS core, (b) PS core with SEBS.

\section{Conclusions}

A classical method of plastic injection molding was converted to a novel method for manufacturing polymermetal hybrid structures in one manufacturing step. Aluminum plates were both deformed and united with polymer during the process which is called polymer injection forming. Plastic injection pressure was very effective in deforming the $\mathrm{Al}$ plates, higher pressures induced tearing of the plate and lower pressures induced insufficient shaping. Optimal injection pressure was found experimentally as $45 \mathrm{MPa}$. The flexural strength and deflection of the polymer-metal hybrid structures were investigated. Three different polymers were used as pure polystyrene and elastomer added polystyrene. Polymeric cores with elastomer material gave better results in bending tests. This shows the success of the PIF process in manufacturing polymer/metal hybrid structures in one manufacturing step by polymer injection forming process.

\section{Acknowledgments}

The authors would like to thank The Scientific and Technological Research Council of Turkey (TUBITAK) for their support in the scope of the research project (Project no: 113M150)

\section{References}

[1] P.F. Bariani, S. Bruschi, A. Ghiotti, G. Lucchetta, CIRP Ann. - Manuf. Technol. 56, 261 (2007).

[2] M. Chen, X. Zhang, Q. Lei, F. Jiexing, Int. J. Mach. Tool. Manuf. 42, 375 (2002).

[3] M. Grujicic, V. Sellappan, M.A. Omar, N. Seyr, J. Mater. Proc. Tech. 197, 363 (2008).

[4] O.J. Zoellner, J.A. Evans, in: ANTEC 2002 Annual Technical Conference, Sanfrancisco, CA 2002, p. 1.

[5] G. Gutscher, H.C. Wu, T. Altan, J. Mater. Proces. Tech. 146, 1 (2004).

[6] F.J. Boerio, P. Shah, J. Adhesion 81, 645 (2005).

[7] H.C.E Van Der Aa, A.S. Verdier, H.G.J De Wolf, WO Patent No. 03057446 (2003).

[8] A.E. Tekkaya, M.M. Hussain, J. Witulski, Prod. Engin. 6, 385 (2012).

[9] D.H. Berry, A. Namkanisorn, J. Adhesion 81, 347 (2005).

[10] H. Sasaki, I Kobayashi, S. Sai, T. Omoto, K. Mori, J. Polym. Tech. 55, 470 (1998). 